\title{
Aplicación de Seis Sigma y los Métodos Taguchi para el Incremento de la Resistencia a la Prueba de Jalón de un Diodo Emisor de Luz
}

Yolanda A. Báez ${ }^{1}$, Jorge Limón ${ }^{1}$, Diego A. Tlapa ${ }^{1}$ y Manuel A. Rodríguez ${ }^{2}$

(1) Universidad Autónoma de Baja California, Facultad de Ingeniería Ensenada, Km. 103 Carretera

Tijuana-Ensenada, Baja California-México (e-mail yolanda@uabc.mx)

(2) Instituto Tecnológico de Ciudad Juárez, Blvd. Tecnológico No. 1340, Ciudad Juárez,

Chihuahua-México

\begin{abstract}
Resumen
El presente trabajo trata sobre la aplicación de la metodología de seis sigma (DMAMC), así como los métodos Taguchi para resolver el problema de baja resistencia a la prueba de jalón de un diodo emisor de luz (LED) de una compañía electrónica del noroeste de México, el cual es utilizado en el ensamble de teléfonos celulares. Al término de este proyecto se logró una mejora significativa al aumentar la capacidad del proceso de 0.56 a 1.45 y un impacto en la reducción de los costos, tan solo por eliminación de desperdicio, del orden de 130 mil dólares estadounidenses anuales. Para lo anterior no se requirió de inversión adicional, únicamente fue necesario controlar el ajuste de cada uno de los factores importantes del proceso.
\end{abstract}

Palabras clave: seis sigma, métodos Taguchi, prueba de jalón, diodo emisor de luz

\section{Implementing Six Sigma and Taguchi Methods to Obtain an Increased Resistance on a Pull Test of a Light Emitting Diode}

\begin{abstract}
This paper deals with the application of six sigma methodology (DMAIC) and Taguchi methods to solve the problem of low resistance of the pulling test of a light emitting diode (LED), which is used in the assembly of cellular telephones in a northwest Mexican electronic company. After finishing this project a significant improvement was achieved that made possible to increase process capability from 0.56 to 1.45 and the impact in cost reduction only by the elimination of waste was of 130 thousand US dollars in savings per year. This achievement did not require additional investment, it was only necessary to control each of the most important factors in the process.
\end{abstract}

Keywords: six sigma, Taguchi methods, pull test, light emitting diode 


\section{INTRODUCCIÓN}

Seis sigma ha sido adoptada en muchas compañías como una nueva estrategia de negocios, la cual se centra en la mejora de los procesos mediante la eliminación de la variación usando una metodología bien estructurada (Bañuelas y Antony, 2004). Esta metodología se originó en Motorola en enero de 1987 como una iniciativa para el mejoramiento de la calidad de largo plazo (Henderson, 2006) y según Li et al. (2008), puede dar como resultado una mayor penetración en el mercado, mayor productividad y costos totales más bajos de manufactura y servicios. El enfoque mayormente utilizado por seis sigma es el método Definir - Medir - Analizar - Mejorar - Controlar (DMAMC), el cual emplea las cartas de control, experimentos diseñados, análisis de capacidad de proceso y muchas otras herramientas estadísticas. Escalante (2003), menciona que Seis Sigma representa una métrica, una filosofía de trabajo y una meta. Como métrica representa una manera de medir el desempeño de un proceso en cuanto a su nivel de productos o servicios fuera de especificación. Como filosofía de trabajo significa mejoramiento continuo de procesos y productos apoyado en la aplicación de la metodología Seis Sigma, la cual incluye principalmente el uso de herramientas estadísticas, además de otras de apoyo. Como meta, un proceso de calidad Seis Sigma significa estadísticamente tener un nivel de clase mundial al no producir servicios o productos defectuosos, ya que solamente se tienen 3.4 unidades defectuosas de cada millón de unidades elaboradas.

Esta metodología, mediante la correcta generación y análisis de datos, promueve el que se dejen de tomar decisiones basadas únicamente en la intuición o presentimientos, para que éstas se conviertan en decisiones basadas en hechos, los cuales surgirán del análisis de los datos. Dados los numerosos casos de éxito conocidos, hoy en día se pueden encontrar bastantes libros y artículos referentes a Seis Sigma. Es importante señalar que el nivel de calidad Seis Sigma no necesariamente se tiene que lograr en todos los procesos de las organizaciones, ya que pueden existir productos o procesos que no impactan de manera significativa en la satisfacción de los clientes y se puede permitir niveles de desempeño menores. Los esfuerzos de esta metodología se enfocan directamente a mejorar lo que es verdaderamente importante para el cliente, incluso en productos conformados por varias dimensiones, Seis Sigma solo mejorará aquellas que puedan considerarse críticas para la calidad (CTQ's) para no desperdiciar esfuerzos.

Un experimento diseñado es extremadamente útil para descubrir las variables claves en los procesos que tienen influencia sobre una característica de calidad de interés (Li et al., 2008). El diseño de experimentos es una técnica estadística poderosa para determinar el ajuste óptimo de los factores que afectan un proceso, para mejorar su funcionamiento, reducir su variabilidad y mejorar la manufactura de productos (Antony y Antony, 2001). Taguchi muestra como el diseño de experimentos estadísticos puede ayudar a los ingenieros a diseñar y manufacturar productos de alta calidad y bajo costo. El poder de los métodos Taguchi es que integra estas técnicas estadísticas dentro de la ingeniería de procesos, por lo que según Li et al., (2005), son ampliamente utilizados para lograr ciertos objetivos en la media de la variable de respuesta mientras simultáneamente se minimiza la varianza.

El concepto de los métodos Taguchi se basa en el diseño de parámetros y la aplicación de las razones señal a ruido. El diseño de parámetros es la clave para lograr bajos costos y alta calidad simultáneamente, ya que durante esta etapa se identifican los factores de control y de ruido, en donde los factores de control se refieren a los parámetros de diseño que se pueden controlar y que serán estudiados a dos o tres niveles durante el diseño experimental, mientras los factores de ruido son aquellos que no se pueden controlar o no se intenta hacerlo. Estos parámetros se organizan en una forma sistemática usando los arreglos ortogonales para conducir el experimento y sus resultados se calculan y comparan usando la razón señal a ruido (Xydas et al., 2005).

Como se mencionó anteriormente uno de los principales objetivos de los métodos Taguchi es lograr producto o procesos robustos, lo que para Wang et al. (2006), significa que el proceso o producto funcione consistentemente y sea relativamente insensible a factores difíciles de controlar, donde según Jong et al. (2006), la estabilidad y la robustez de un producto mejoran conforme se incrementa la razón señal a ruido, ya que como menciona Palanikumar (2006), las fórmulas de la razón señal a 
ruido están diseñadas de tal forma que el experimentador siempre escoja la combinación de niveles de los factores que resulten en el mayor valor de esta razón para optimizar la característica de calidad de un experimento. Por lo tanto el método para calcular la razón señal a ruido depende de si la característica de calidad es del tipo lo menor es lo mejor, lo mayor es lo mejor o lo nominal es lo mejor.

El objetivo del presente trabajo es la aplicación de la metodología Seis Sigma y los métodos Taguchi para atacar el problema de la falta de resistencia en el ensamble de un Diodo Emisor de Luz (LED) al momento de ser soldados en las tarjetas de circuito impreso (PCB) del cliente. Para realizar la prueba de jalón, se utilizó un instrumento en el cual se coloca la pieza para ser destruida por medio de una cuña que mide la presión utilizada para desprender el LED de la carcasa (Housing), obteniendo así la resistencia de la unidad. Se piensa que la falta de resistencia es debida a la inestabilidad en el proceso, consecuencia del desconocimiento y la falta de control de los factores que intervienen en él, ya que éstos son controlados a prueba y error por supervisores y/o operadores, lo cual provoca que las unidades de este producto presenten una marcada diferencia en cuanto a resistencia en dicha prueba, dando como resultado algunas piezas que no cumplen con la especificación mínima de 5 libras especificada por la empresa, lo cual provoca quejas posteriores, debido al desprendimiento del diodo de la tarjeta PCB durante el proceso de ensamble realizado por el cliente. Este producto representa el $40 \%$ de las ventas de la empresa por ser el artículo principal de un importante contrato con una conocida empresa que manufactura celulares.

\section{METODOLOGÍA}

La metodología empleada en este trabajo es la utilizada en la filosofía seis sigma, por ser un esquema que se utiliza frecuentemente en la actualidad para lograr el mejoramiento continuo de la calidad de los procesos. Esta metodología consta de cinco fases las cuales son definir, medir, analizar, mejorar y controlar, mismas que irán apareciendo en el desarrollo de este trabajo de acuerdo al orden en que se utilizaron.

\section{Fase definir}

Por tener un efecto crítico en la calidad del producto se determinó que la característica considerada para el estudio es la resistencia a la prueba de jalón. Además, según se muestra en la Figura 1, los defectos de este tipo representan el segundo defecto en importancia. Cabe mencionar que para el defecto de coplanaridad, que es el primero en importancia los autores realizaron un proyecto alterno en el que se logró reducir significativamente. En esta fase también se definió como objetivo aumentar el índice $C_{p k}$ a un mínimo de 1.33 .

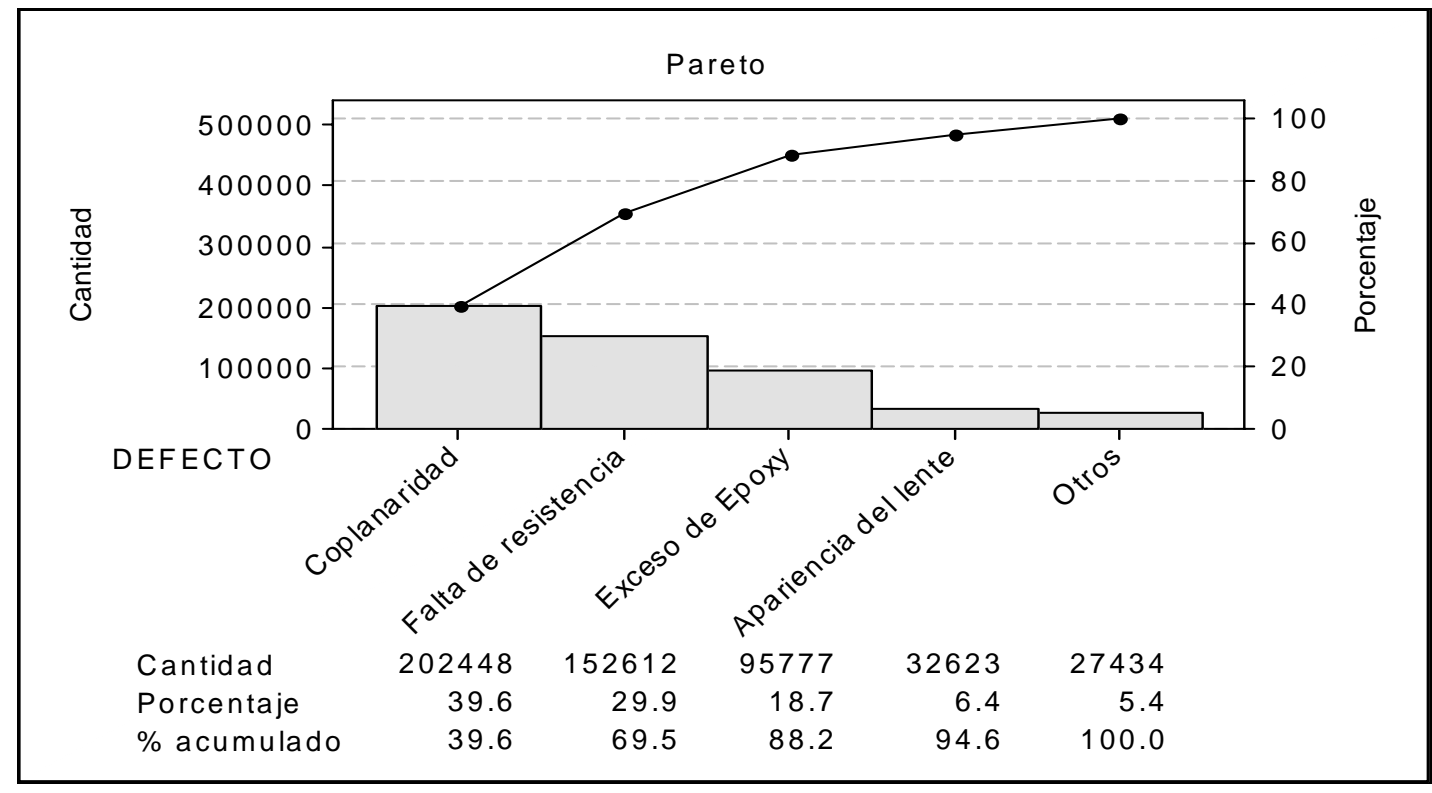

Fig. 1: Diagrama de Pareto de los defectos de la línea de producción analizada. 
Fase medir

El siguiente paso fue determinar el comportamiento del proceso de acuerdo a los requerimientos de resistencia del producto siendo ésta una característica crítica para la calidad del estudio (CTQ), por lo que fue esencial establecer la precisión del sistema de medición y la calidad de los datos obtenidos. Con este objetivo se realizó un estudio de repetibilidad y reproducibilidad (RyR) el cual se utiliza para identificar las fuentes de variación que contribuyen a la variación total en las mediciones obtenidas por algún sistema de medición y como una consecuencia, evaluar su poder de discriminación (Henderson, 2006). Hecho el análisis puede ser necesario rediseñar el instrumento, revisar si sus componentes están gastados o sucios, si la repetibilidad es la fuente dominante de variación. Cuando la reproducibilidad es la mayor fuente de variación, los esfuerzos se deberán enfocar en estandarizar los procedimientos de medición y entrenar a los operadores para que se apeguen a ellos. Un sistema de medición se considera aceptable cuando su variabilidad es menor del 10 por ciento de la variabilidad total del proceso, puede ser aceptable dependiendo de la importancia del producto o los costos si representa entre el 10 y 30 por ciento y si representa más del $30 \%$ se considera no aceptable (AIAG, 2002). Este estudio de repetibilidad y reproducibilidad mostró que el sistema de medición representa el 4.06\%, lo que implica que es aceptable, además de que el número de categorías distintas fue de 6 , siendo 5 el mínimo recomendado (AIAG, 2002). La Tabla 1 muestra los resultados obtenidos en el estudio R\&R llevado a cabo en la fase de medición del proyecto.

Tabla 1: Resultados obtenidos al realizar la prueba R\&R

\begin{tabular}{lcc}
\hline \multicolumn{3}{c}{ Análisis de componentes de variación } \\
\hline Componentes de variación & Desviación estándar & Contribución a la variación \\
\hline Total Gage R\&R & 0.18 & 4.06 \\
Repetibilidad & 0.18 & 4.06 \\
Reproducibilidad & 0.00 & 0.00 \\
Parte a Parte & 4.27 & 95.94 \\
Variación Total & 4.45 & 100.00 \\
Número de categorías diferentes $=6$ & \\
\hline
\end{tabular}

La línea base de la capacidad del proceso $\left(C_{p k}\right)$ también se obtuvo en esta fase una vez que se determinó que las mediciones eran confiables. De esta forma el estudio de capacidad del proceso se llevó a cabo recabando 25 muestras de tamaño 4 cada una, durante 5 días en los diferentes turnos.

Tabla 2: Datos de las resistencias de productos fabricados utilizando el ajuste actual del proceso

\begin{tabular}{ccccc|ccccc}
\hline \multicolumn{1}{l}{ Subgrupo } & \multicolumn{10}{c}{ Subgrupo } \\
\hline 1 & 6.81 & 11.20 & 15.52 & 10.55 & 14 & 10.67 & 11.53 & 11.59 & 11.50 \\
2 & 13.23 & 11.23 & 8.12 & 9.81 & 15 & 8.29 & 8.26 & 7.24 & 6.13 \\
3 & 5.85 & 10.25 & 11.17 & 13.30 & 16 & 15.17 & 13.94 & 5.83 & 4.73 \\
4 & 11.26 & 11.06 & 8.19 & 9.57 & 17 & 10.85 & 9.65 & 11.53 & 10.56 \\
5 & 3.56 & 10.54 & 14.93 & 11.60 & 18 & 10.27 & 14.96 & 7.60 & 7.49 \\
6 & 10.26 & 9.50 & 8.61 & 4.91 & 19 & 10.15 & 10.27 & 11.21 & 12.40 \\
7 & 13.30 & 13.28 & 5.47 & 17.54 & 20 & 8.59 & 8.68 & 8.21 & 5.22 \\
8 & 8.70 & 8.34 & 11.24 & 10.09 & 21 & 6.40 & 15.63 & 9.57 & 7.50 \\
9 & 8.60 & 13.54 & 13.29 & 9.72 & 22 & 6.54 & 8.20 & 7.27 & 9.38 \\
10 & 14.39 & 4.43 & 2.72 & 11.68 & 23 & 15.15 & 3.13 & 7.90 & 11.63 \\
11 & 9.68 & 13.44 & 2.39 & 13.01 & 24 & 8.69 & 8.89 & 6.09 & 8.77 \\
12 & 9.60 & 6.96 & 12.71 & 7.00 & 25 & 15.55 & 12.51 & 11.03 & 11.10 \\
13 & 11.66 & 13.04 & 11.62 & 11.72 & & & & & \\
\hline
\end{tabular}

La Tabla 2 muestra la información obtenida y el análisis efectuado con Minitab 14, se observa en la Figura 2, en la que se puede apreciar mediante las cartas de control $\bar{X}$ y $R$ que el proceso es estable a través del tiempo, asimismo mediante el histograma y la prueba Anderson Darling (AD) se puede 
apreciar que los datos siguen una distribución normal, sin embargo el índice de capacidad $C_{p k}=0.56$ lo cual es demasiado bajo considerando que la característica de calidad es considerada crítica, por lo que es claramente evidente la necesidad de mejorar éste proceso. Cabe aclarar que el índice de capacidad $C_{y}$ no se puede calcular dado que la característica de calidad solo tiene límite de especificación inferior.

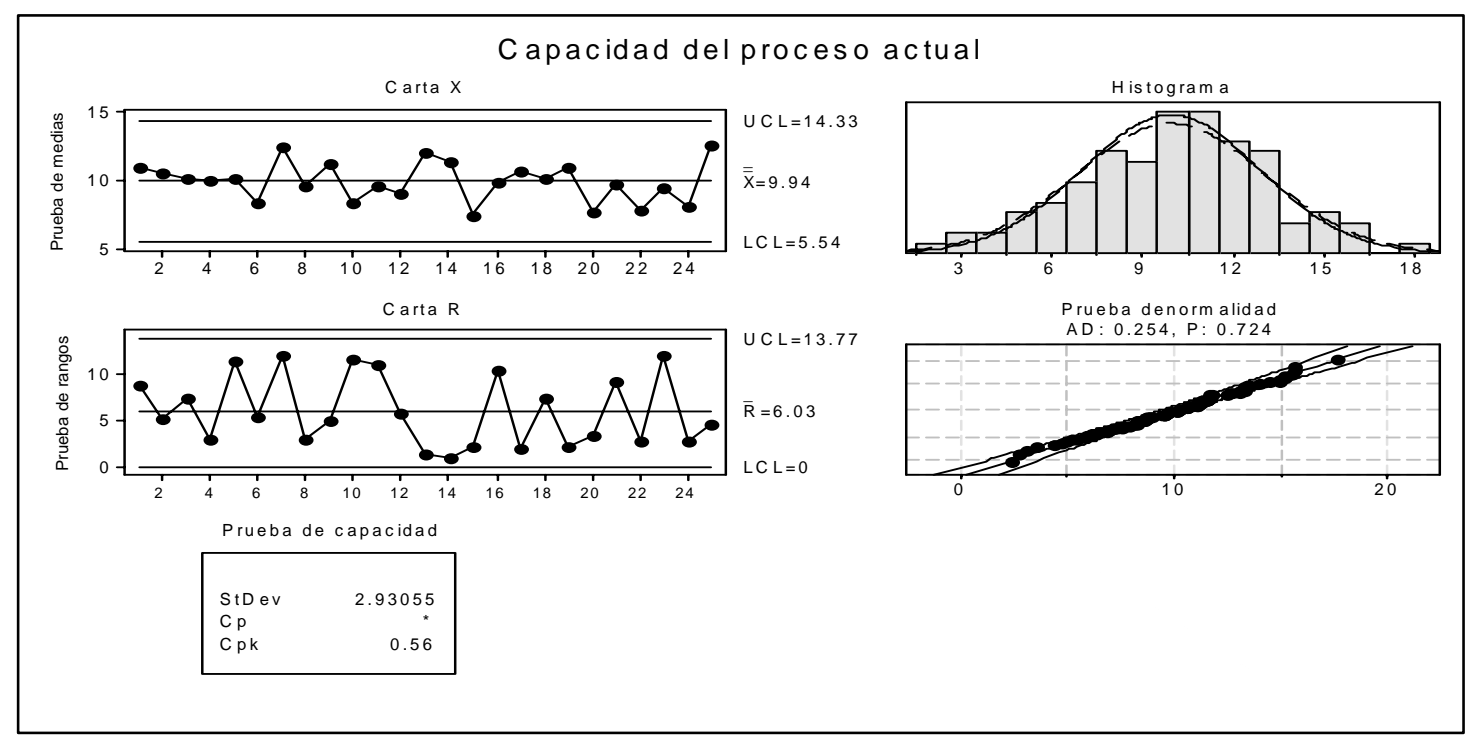

Fig. 2: Gráficas de la capacidad del proceso en el que se utiliza el ajuste de factores inicial

\section{Fase analizar}

En esta fase se realizó un análisis completo del proceso y se construyó un diagrama causa-efecto mediante una lluvia de ideas del equipo de trabajo para identificar las variables que pudieran estar afectando la resistencia a la prueba del jalón del producto, el cual se muestra en la Figura 3. Mediante un análisis más a fondo con las causas posibles y con algunas corridas de prueba se concluyó que las variables para considerar en estudios posteriores serían las que se muestran en la Tabla 3.

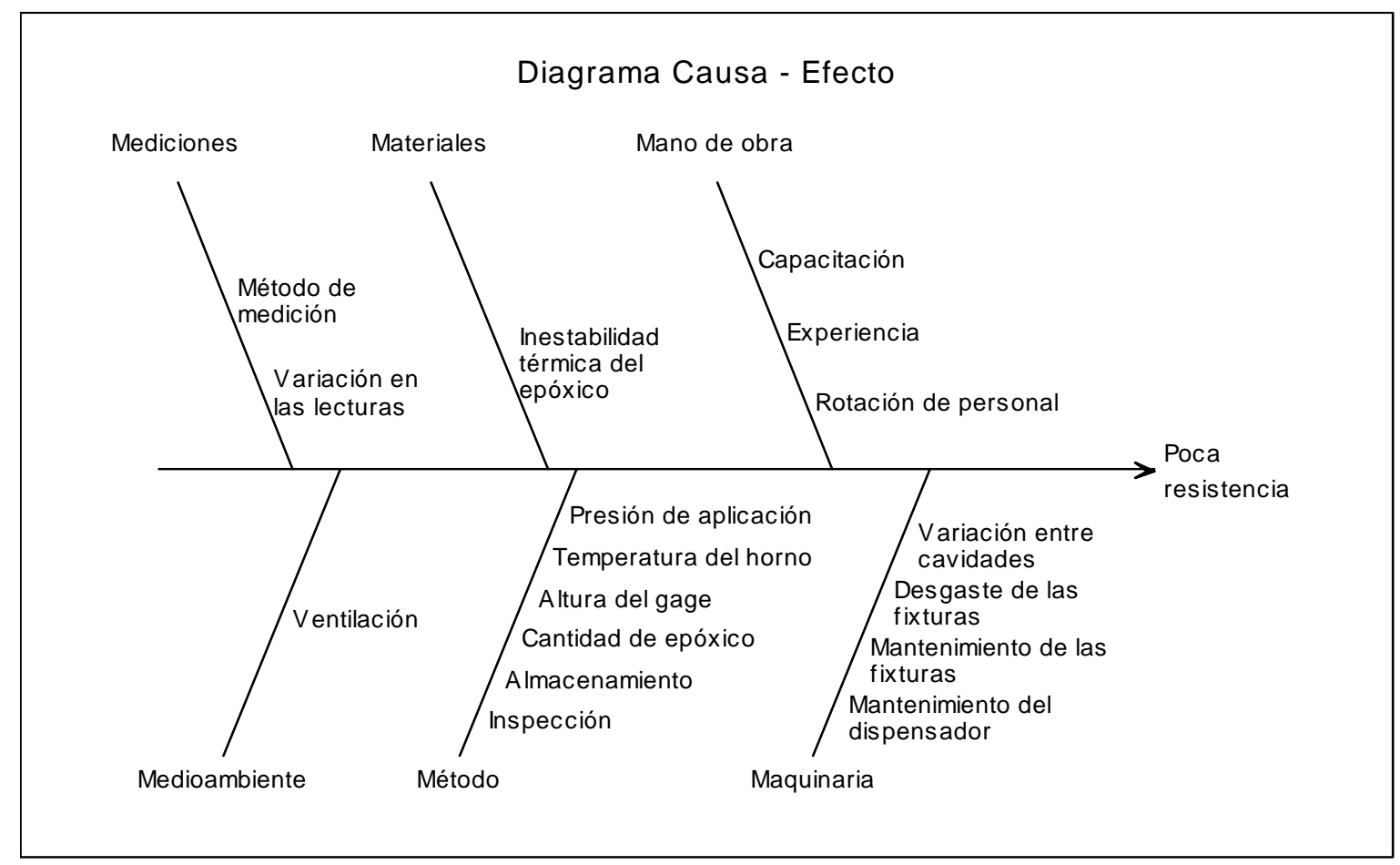

Fig. 3: Diagrama Causa - Efecto de los posibles factores que afectar la resistencia del producto 
Tabla 3: Variables consideradas

\begin{tabular}{lc}
\hline & Variables \\
\hline Cantidad de epóxico & Vida del epoxi \\
Presión de aplicación & Desgaste de los moldes \\
Ajuste de altura & Diferencia entre cavidades \\
Ventiladores & Temperatura \\
\hline
\end{tabular}

Posteriormente se da una breve descripción de los factores y el porqué se consideran en el estudio. Es en esta fase también en la que se decide utilizar los métodos Taguchi para optimizar el proceso.

Cantidad de epóxico. Se probó a cuatro niveles, 100, 75, 50 y 25 \% de aplicación y se eligió de esta manera porque había interés en conocer si con menos del 100\% de aplicación el producto alcanzaría la resistencia necesaria.

Presión de aplicación. Este factor se evaluó a dos niveles, el primer nivel es el que normalmente se trabaja, rango de 30-40 psi, y el segundo nivel 50-60 psi, ya que previamente se estudió arrojando buenos resultados.

Ajuste de altura. El dado ajustador que sostiene al dispensador del epóxico puede ajustarse a dos niveles de altura preestablecidos. Para finalidad del experimento, el primer nivel es ajuste alto (utilizado normalmente) y el segundo nivel es ajuste bajo, en este nivel las agujas de dispensador quedan más cerca del molde.

Ventiladores. Los ventiladores que se encuentran en el área de trabajo se consideran un factor que afecta al proceso, por argumentar que el viento enfría el horno provocando que las piezas no curen correctamente. Para comprobar su influencia se estudian a dos niveles: encendidos y apagados.

Escalera. Por escalera se entiende las temperaturas a que se encuentran las cinco fases por las que pasa el producto dentro del horno. Para efectos del experimento, en el primer nivel se considera la escalera que se utiliza normalmente en el proceso, y como segundo nivel se incrementa la temperatura $15^{\circ} \mathrm{C}$ en cada fase, por considerar que temperaturas más altas darían mejor resultado, cabe mencionar que el aumento se consideró según la experiencia del personal involucrado y algunas pruebas preliminares, ya que existe el riesgo de quemar el epóxico por un inadecuado incremento de temperaturas.

Vida del epóxico. Se conoce como tiempo máximo de exposición de la resina epóxica a temperatura ambiente recomendado por el proveedor para asegurar en éste las características apropiadas para ser usado en el proceso estudiado. Durante sus cuatro horas de vida útil, la resina sufrirá pequeños cambios en su temperatura, lo que traerá como consecuencia incremento de viscosidad, provocando que las agujas del dispensador se tapen, depositando diferentes cantidades de resina entre productos, lo que representaría diferencias en la resistencia de los productos a la prueba de jalón.

Desgaste del molde. El estado de los moldes de ensamble del LED es un factor importante ya que puede afectar en la resistencia final, por lo que se deberá fijar un proceso que sea mínimamente afectado por el desgaste del molde durante su vida útil. El experimento se corrió en dos moldes con tapa, seleccionando uno nuevo y otro con nivel considerable de desgaste.

Diferencia entre cavidades. Otro factor considerado en el experimento es la diferencia que existe entre las cavidades de un mismo molde. Se integra al experimento, por considerar que pudiera afectar los resultados en la resistencia a la prueba de jalón. Después de pruebas previas, mediante análisis de varianza, se determinó que para fines del experimento se utilizarán las cavidades 3 y 12.

La vida del epóxico, el desgaste de los moldes y la diferencia entre cavidades se consideran como factores de ruido, ya que resulta impráctico o antieconómico controlarlos pero de alguna manera pueden influir en resistencia del producto, por lo que se debe encontrar la condición de operación del proceso que sea mínimamente afectada por dichos factores. 


\section{Fase de mejorar}

En esta etapa se corrió el experimento utilizando los métodos Taguchi como se especificó anteriormente, debido a que estos requieren un reducido número de corridas experimentales, ya que como lo explica Cesarone (2001), con ellos se prueba solo una pequeña fracción de todas las combinaciones posibles de los factores, de tal manera que permitan calcular los efectos de todas las entradas sobre las salidas. Para esto, primeramente los factores se dividieron en factores de control y de ruido. Los factores estudiados tanto de control como de ruido, así como sus respectivos niveles se muestran en la Tabla 4.

Tabla 4: Factores y sus niveles

Factores de control
A) Cantidad de epóxico
B) Presión de aplicación
C) Ajuste de altura
D) Ventiladores
E) Temperatura

Factores de ruido

1) Vida del epóxico

2) Desgaste de los moldes

3) Diferencia entre cavidades

$\begin{array}{cc}\text { Nivel 1 } & \text { Nivel } 2 \\ 100 \% & 75 \% \\ 30-40 \text { psi } & 50-60 \text { psi } \\ \text { dado alto } & \text { dado bajo } \\ \text { encendido } & \text { apagado } \\ 6{ }^{\circ} \mathrm{C}, 300{ }^{\circ} \mathrm{C}, 294{ }^{\circ} \mathrm{C}, 170{ }^{\circ} \mathrm{C} \\ 1{ }^{\circ} \mathrm{C}, 315{ }^{\circ} \mathrm{C}, 309{ }^{\circ} \mathrm{C} & 185{ }^{\circ} \mathrm{C} \\ \text { Nivel } 1 & \\ \text { cién salido del refrigerador } \\ \text { molde Nuevo } \\ \text { cavidad 3 }\end{array}$

$50-60$ psi

dado bajo

Nivel 1: $306{ }^{\circ} \mathrm{C}, 300{ }^{\circ} \mathrm{C}, 294{ }^{\circ} \mathrm{C}, 170{ }^{\circ} \mathrm{C}$ y $70{ }^{\circ} \mathrm{C}$

Nivel 2: $321{ }^{\circ} \mathrm{C}, 315{ }^{\circ} \mathrm{C}, 309^{\circ} \mathrm{C} 185^{\circ} \mathrm{C}$ y $85^{\circ} \mathrm{C}$

recién salido del refrigerador cavidad 3

$\begin{array}{cc}\text { Nivel 3 } & \text { Nivel } 4 \\ 50 \% & 25 \% \\ ---- & ---- \\ ---- & ---- \\ ---- & --- \\ & \end{array}$

Nivel 2

2 horas en operación molde desgastado cavidad 12

Para albergar estos factores fue necesaria la utilización de dos Arreglos Ortogonales (AO), un $\mathrm{L}_{8}$ para el arreglo interno y un $\mathrm{L}_{4}$ para el arreglo externo. Son estos $A O$ los que permiten realizar la experimentación con un número reducido de corridas ya que son arreglos factoriales altamente fraccionados. Una consecuencia lógica de hacer solo una fracción de las combinaciones posibles es la pérdida de información, ya que algunos efectos se consideran de antemano como no importantes como lo son los efectos de algunas o todas las interacciones posibles, lo cual puede ser un factor de riesgo, debido a que una interacción inesperada entre dos o más parámetros podría alterar de una manera importante las conclusiones del análisis (Goh, 1993). Para este experimento se realizó una modificación al arreglo interno para poder satisfacer los requerimientos del experimento, ya que el factor llenado del epóxico se probó a cuatro niveles $\left(A_{1}, A_{2}, A_{3}, A_{4}\right)$. Para los factores de ruido fue necesario el $\mathrm{AO} \mathrm{L}_{4}$, el cual permite estudiar tres factores a dos niveles. En la Tabla 5 se muestra la matriz de diseño, resultado de la combinación de la matriz interna $L_{8}$ (modificada) y la matriz externa $\mathrm{L}_{4}$.

Tabla 5: Matriz de diseño

\begin{tabular}{|c|c|c|c|c|c|c|c|c|c|c|}
\hline & \multicolumn{4}{|c|}{ Factores } & 3 & 1 & 2 & 2 & 1 & \multirow{2}{*}{$\begin{array}{l}\text { Arreglo } \\
\text { externo }\end{array}$} \\
\hline & \multicolumn{2}{|c|}{ Arreglo } & \multicolumn{2}{|c|}{ nterno } & 2 & 1 & 2 & 1 & 2 & \\
\hline & & & & & & & & & & \\
\hline Corrida & A & B & C & D & $E$ & & & & & \\
\hline 1 & 1 & 1 & 1 & 1 & 1 & & & & & \\
\hline 2 & 1 & 2 & 2 & 2 & 2 & & & & & \\
\hline 3 & 2 & 1 & 1 & 2 & 2 & & & & & \\
\hline 4 & 2 & 2 & 2 & 1 & 1 & & & & & \\
\hline 5 & 3 & 1 & 2 & 1 & 2 & & & & & \\
\hline 6 & 3 & 2 & 1 & 2 & 1 & & & & & \\
\hline 7 & 4 & 1 & 2 & 2 & 1 & & & & & \\
\hline 8 & 4 & 2 & 1 & 1 & 2 & & & & & \\
\hline
\end{tabular}

La simbología $y_{i j}$ representa a los valores resultantes al correr el experimento, en cada uno de los cruces entre los niveles de los factores de control y niveles de los factores de ruido, descritos con anterioridad. 
En esta etapa también se corrió el experimento teniendo la precaución de cuidar cada uno de los detalles que pudieran afectar el resultado de los experimentos y se realizaron las corridas de acuerdo al orden del arreglo resultante. Primero se realizaron las corridas que correspondían a la cantidad de epóxico al $100 \%$, posteriormente las de llenado al $75 \%, 50 \%$ y $25 \%$ respectivamente, ya que este factor fue ubicado en la primera columna del arreglo interno. No fue posible aleatorizar el orden de las corridas, puesto que el llenado de epóxico se realiza mediante un dispensador, el cual se tuvo que ir modificando para la prueba, es decir, se le fueron cerrando las agujas de acuerdo al nivel de llenado requerido, por lo que al final de la experimentación el dispositivo quedó destruido. Por lo costoso y escaso del dispensador se optó por hacerlo de esta manera, esto atendiendo a lo mencionado por De la vara (2004), quien argumenta que Taguchi no enfatiza la necesidad de correr el experimento en orden aleatorio como se recomienda en el diseño clásico, sino mas bien presupone las complicaciones prácticas del proceso y estructura el orden de las columnas conforme a estas dificultades. Una vez realizadas todas las corridas, se aleatorizó completamente el orden en que se realizaron las pruebas de jalón a las piezas, utilizando el mismo equipo de medición y realizadas por el mismo operador, todo lo anterior con la idea de evitar cualquier alteración en los datos.

El siguiente paso del experimento consiste en analizar los datos obtenidos en las corridas, y contenidos en la matriz de diseño, con la finalidad de evaluar que factores afectan la variación de los datos, y los niveles a los que deberán ser fijados para establecer la configuración final del proceso. La variación es evaluada respecto a la media de los datos y al estadístico señal a ruido, el cual mide el desempeño de la robustez en cada combinación de los factores de control (De la vara, 2004).

El estudio tiene como objetivo maximizar la resistencia a la prueba de jalón del producto de la línea PRISMA I, por lo que la variable de respuesta (característica de calidad), es del tipo "Lo mayor es lo mejor", y le corresponde la siguiente transformación de señal a ruido:

$\eta d B=-10 \log \left[\frac{1}{n} \sum_{i=1}^{n} \frac{1}{Y_{i}^{2}}\right]$

En la Tabla 6, se muestra el arreglo completo con los cálculos de la media (promedio), y señal a ruido para analizar la resistencia a la prueba de jalón.

Tabla 6: Cálculo de la razón señal a ruido

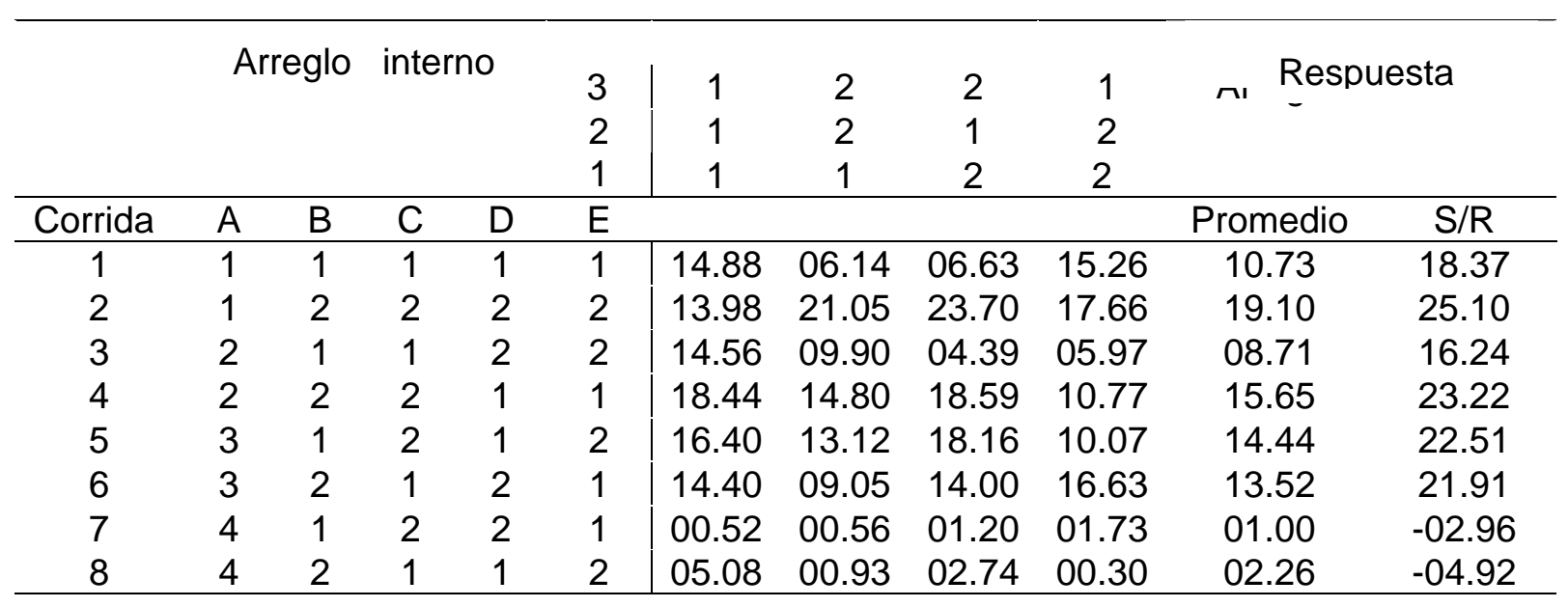

Una vez obtenidos los resultados mostrados anteriormente, se procede a construir las gráficas factoriales para la razón señal a ruido y los promedios, respectivamente, los cuales se obtuvieron utilizando el software Minitab 14. La gráfica factorial para la variable de respuesta natural y para la razón señal a ruido se muestran en las Figuras 4 y 5 respectivamente. Del análisis de estas gráficas, se como deduce como la mejor combinación de los factores de control: $A_{3} B_{2} C_{2}$ para la razón señal a ruido y $A_{1} B_{2} C_{2}$ para los promedios. La pendiente casi nula para los factores $D$ y $E$ sugiere que el efecto de estas variables no es significativo para ambas respuestas. 


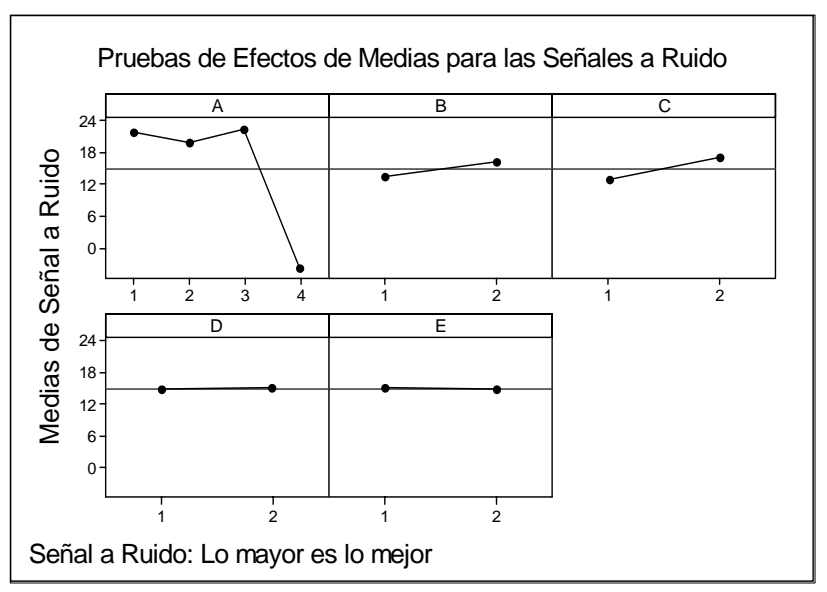

Fig. 4 Efectos de medias para la Señal a Ruido

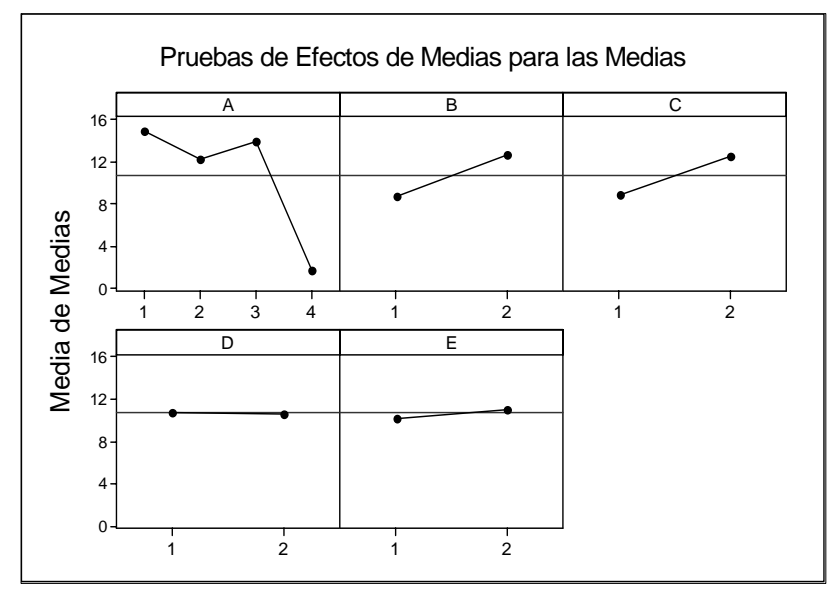

Fig. 5 Efectos de medias para la media

Posteriormente se efectuó el análisis de varianza (ANOVA) para verificar estadísticamente el efecto de los factores tanto en la razón señal a ruido (Tabla 7), como en la resistencia promedio (Tabla 8). En este análisis se comprueba que las variables $D$ y $E$ no son significativas, por lo se agrupan al error aleatorio, para obtener el error aleatorio agrupado, que se usó en los cálculos posteriores.

Tabla 7: Análisis de varianza para la razón señal a ruido de los datos transformados

\begin{tabular}{cccccc}
\hline Factores & Grados de libertad & Suma de cuadrados & Cuadrados medios & $F$ & $P$ \\
\hline $\mathrm{A}$ & 3 & 957.69 & 319.23 & 1329.05 & 0.001 \\
$\mathrm{~B}$ & 1 & 15.50 & 15.50 & 64.52 & 0.015 \\
$\mathrm{C}$ & 1 & 33.06 & 33.06 & 137.65 & 0.007 \\
${ }^{*} \mathrm{D}$ & 1 & 0.15 & ----- & ----- & ----- \\
${ }^{*} \mathrm{E}$ & 1 & 0.33 & ---- & ---- & ---- \\
\hline Error & -------- & --- & ---- \\
\hline (Error) & 2 & ---- & 0.24 & -- & - \\
\hline Total & 7 & 0.48 & & & \\
\hline
\end{tabular}

Tabla 8: Análisis de varianza para la media (Datos sin transformar)

\begin{tabular}{cccccc}
\hline Factores & Grados de libertad & Sumas de cuadrados & Cuadrados medios & $\mathrm{F}$ & $\mathrm{P}$ \\
\hline $\mathrm{A}$ & 3 & 903.16 & 301.05 & 22.89 & 0.000 \\
$\mathrm{~B}$ & 1 & 122.58 & 122.58 & 9.32 & 0.005 \\
$\mathrm{C}$ & 1 & 112.09 & 112.09 & 8.52 & 0.008 \\
${ }^{*} \mathrm{D}$ & 1 & 0.28 & 0.28 & 0.02 & 0.885 \\
${ }^{*} \mathrm{E}$ & 1 & 6.49 & 6.49 & 0.49 & 0.849 \\
Error & 24 & 315.61 & 13.15 & ---- & ---- \\
\hline (Error) & 26 & 322.38 & 12.40 & ---- & ---- \\
\hline Total & 31 & 1460.21 & & & \\
\hline
\end{tabular}

Una vez efectuado el análisis de varianza se obtuvo como resultado que los factores que afectan significativamente ambas variables de respuesta son: cantidad de epóxico, presión de aplicación y altura del dado, por lo que es importante que éstos se fijen a sus mejores niveles. Los factores $D$ y $E$ resultaron no significativos por lo que se deben trabajar como normalmente se hace o como resulte más económico o más fácil para la empresa. Debido a que estos factores presentan sumas de cuadrados pequeñas son agrupados al error aleatorio, lo cual permite realizar un análisis de varianza para la razón señal a ruido en el que se pueda tener una estimación del error aleatorio, mostrando que los factores significativos para esta variable transformada son los mismos que para la respuesta sin transformar. Mediante el análisis de varianza y con el apoyo de las gráficas factoriales se obtienen los factores significativos a sus mejores niveles, quedando de la siguiente manera: $A_{1}$ ) Cantidad de epóxico:100\% $B_{2}$ ) Presión de aplicación: 50-60 psi $C_{2}$ ) Ajuste de altura: baja. 
Posteriormente se llevó a cabo la predicción del funcionamiento del proceso con los niveles óptimos. Para la elaboración del pronóstico del valor esperado de respuesta, se consideran únicamente los efectos principales que afectan a la variable de salida, es decir, el ajuste propuesto del proceso: $\begin{array}{lll}A_{1} & B_{2} & C_{2}\end{array}$, mediante la siguiente ecuación de predicción para la resistencia a la prueba de jalón:

$$
y=\bar{y}+\left(\bar{A}_{1}-\bar{y}\right)+\left(\bar{B}_{1}-\bar{y}\right)+\left(\bar{C}_{1}-\bar{y}\right)
$$

Predicción para la media a la resistencia de la prueba de jalón

$\hat{\varphi}=10.676+(14.91-10.67)+(12.63-10.67)+(12.54-10.67)=18.74$ libras estimadas

Predicción para la S/R de la resistencia de la prueba de jalón

$\hat{y}=14.93+(21.74-14.93)+(16.32-14.93)+(16.97-14.93)$

$$
\hat{y}=25.16 d B
$$

Cabe señalar que los factores y sus niveles indicados coinciden con la corrida dos del arreglo interno y los valores predichos son muy cercanos a los obtenidos en el experimento, para la señal a ruido se predijo $25.16 \mathrm{~dB}$ y se obtuvo $25.10 \mathrm{~dB}$; mientras que para la media se predijo $18.74 \mathrm{lb}$ y se obtuvo $19.10 \mathrm{lb}$. Considerando que los niveles óptimos se encuentran en una corrida del experimento, no es necesario realizar la corrida de confirmación, sin embargo ésta se realizó para reforzar la decisión. Esta corrida consistió en tomar las piezas de un molde con cada uno de los ajustes (inicial y propuesto), teniendo un total de 25 muestras con cada uno, los datos se muestran en la Tabla 9.

Tabla 9: Lecturas de la resistencia a la prueba de jalón

\begin{tabular}{c|c|c|c|c|c|c}
\hline \multicolumn{2}{c|}{ AJUSTE PROPUESTO $X_{1 j}$} & \multicolumn{3}{|c|}{ AJUSTE INICAL $X_{2 j}$} & \multicolumn{2}{c}{ DIFERENCIA } \\
$D_{j}=X_{1 j}-X_{2 j}$
\end{tabular}

Posteriormente, se realizó una comparación t-pareada (Tabla 10) en la que se obtiene que la media de la resistencia del ajuste propuesto es mejor estadísticamente, la diferencia de medias se encuentra, con un 95\% de confianza, en el intervalo formado por los valores 5.51266 y 8.61294 . 
Tabla 10: Resultados de la t-pareada

\begin{tabular}{lccc}
\hline Ajuste de factores & $\mathrm{N}$ & Media & Desv. estándar \\
\hline Propuesto & 25 & 17.32 & 2.82 \\
Inicial & 25 & 10.25 & 2.60 \\
Diferencia & 25 & 7.06 & 3.76 \\
\hline
\end{tabular}

Antes de efectuar la prueba t-pareada, se procedió a comprobar el supuesto de que las diferencias $D_{i}=x_{1 i}-x_{2 j}$ siguen una distribución normal, para lo cual se llevó a cabo la prueba AndersonDarling (Figura 6) en la cual se obtuvo un P-Value de 0.358 por lo cual no se rechaza la hipótesis de normalidad de los datos.

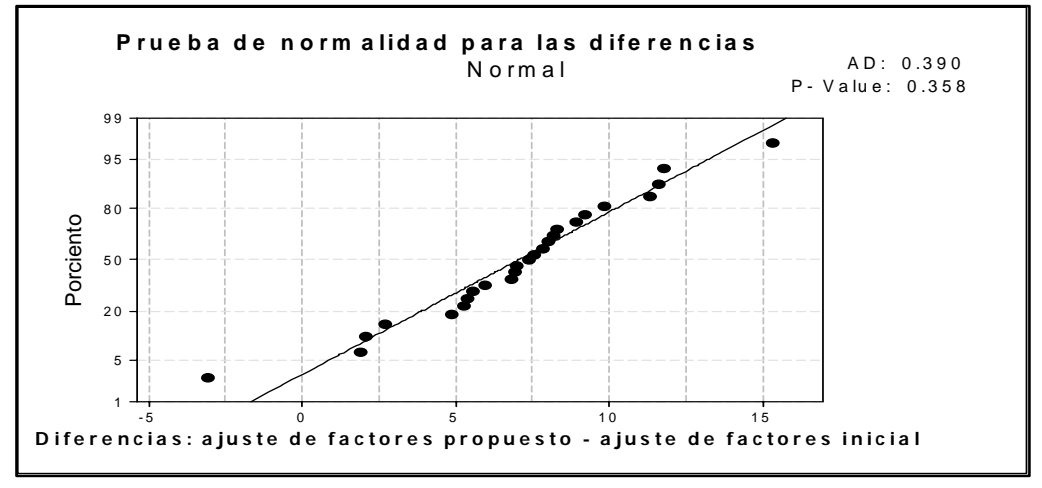

Fig. 6: Prueba de normalidad para las diferencias

\section{Fase controlar}

El gran reto de la metodología Seis Sigma quizás no sea la mejora de los procesos, sino mantener los resultados óptimos durante su operación cotidiana. Esto requiere de la estandarización de los métodos de trabajo y de un monitoreo y control de forma continua de las operaciones. En esta ocasión para lograr este fin, una vez validada la mejora se procedió a cambiar las condiciones de operación de este proceso conforme a las condiciones óptimas encontradas en la fase anterior del proyecto, y a la construcción de cartas de control, para que los operadores puedan tomar acciones preventivas antes de que estos parámetros evaluados empiecen a salirse de control con la consecuente pérdida de calidad. Para el establecimiento de los límites de control del proceso se recolectaron 25 muestras de tamaño 4 cada una durante 5 días en los diferentes turnos, los datos se muestran en la Tabla 11.

Tabla 11: Muestras recolectadas para los límites de control del proceso utilizando el ajuste propuesto

\begin{tabular}{ccccc|ccccc}
\hline Subgrupo & \multicolumn{7}{c}{ Subgrupo } \\
\hline 1 & 13.31 & 16.96 & 19.39 & 17.66 & 14 & 15.41 & 16.62 & 14.46 & 21.24 \\
2 & 10.17 & 14.06 & 14.92 & 14.62 & 15 & 20.54 & 18.65 & 19.74 & 14.92 \\
3 & 17.55 & 16.13 & 15.76 & 18.81 & 16 & 19.69 & 12.24 & 14.69 & 21.66 \\
4 & 9.93 & 15.04 & 15.23 & 17.63 & 17 & 21.26 & 16.99 & 20.50 & 18.72 \\
5 & 20.84 & 16.09 & 13.62 & 18.87 & 18 & 18.82 & 17.74 & 16.69 & 20.06 \\
6 & 22.20 & 16.87 & 21.51 & 15.70 & 19 & 14.92 & 15.85 & 17.40 & 16.14 \\
7 & 19.89 & 20.41 & 19.34 & 17.48 & 20 & 13.17 & 15.31 & 18.59 & 12.49 \\
8 & 12.03 & 18.90 & 14.23 & 17.35 & 21 & 16.05 & 19.13 & 20.80 & 22.42 \\
9 & 17.75 & 23.74 & 16.82 & 12.56 & 22 & 17.84 & 12.67 & 19.88 & 15.35 \\
10 & 15.29 & 16.84 & 14.14 & 13.74 & 23 & 12.64 & 19.44 & 16.45 & 19.28 \\
11 & 18.85 & 18.40 & 17.21 & 21.64 & 24 & 15.65 & 13.37 & 15.51 & 18.17 \\
12 & 14.00 & 12.04 & 18.49 & 21.78 & 25 & 18.47 & 17.75 & 15.67 & 16.16 \\
13 & 17.82 & 21.08 & 21.08 & 18.13 & & & & & \\
\hline
\end{tabular}

Con esta información se procedió al cálculo del índice $C_{p k}$, los PPM's y el nivel de sigmas del proceso para poder comparar con el proceso inicial (Figura 7). 


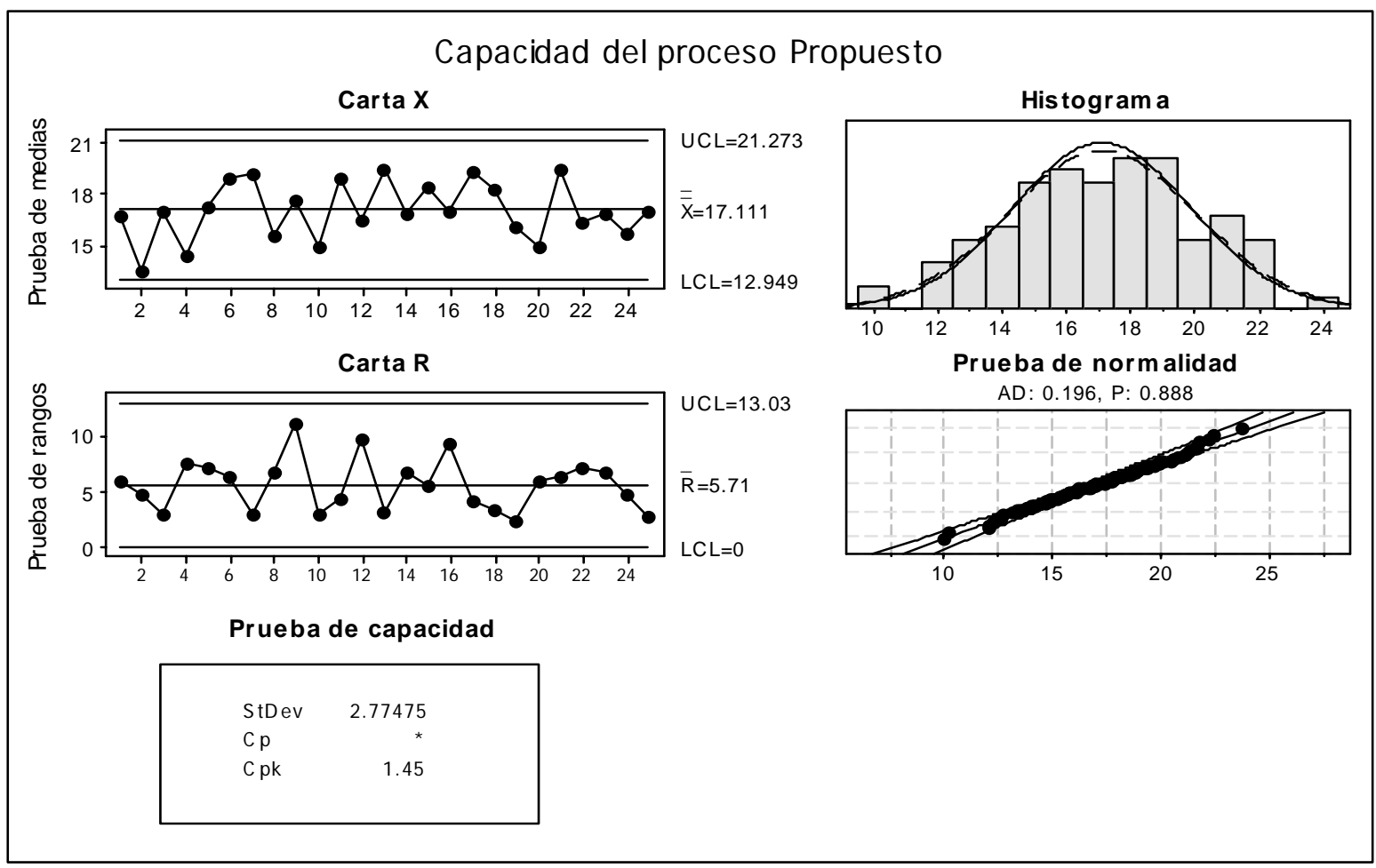

Fig. 6: Cartas de control para el proceso propuesto

\section{ANÁLISIS DE RESULTADOS}

La línea de producción donde se desarrolló el proyecto trabaja 18 horas al día, durante cinco días a la semana, dando como resultado una producción de 1'170,000 unidades mensuales, información con la cual se proyecta una producción aproximada de 14040,000 unidades anuales.

El costo unitario del producto, se determinó considerando únicamente los costos directos de producción, como lo son materia prima, mano de obra e insumos, dando como resultado un costo de 20 centavos de dólar estadounidense por unidad.

Para determinar el ahorro generado por el proyecto se consideran los datos descritos anteriormente, por lo que no se encuentran incluidos los gastos por retornos de garantía del cliente, es decir, solo se considera el costo generado por una unidad defectuosa detectada dentro de la compañía. En la Tabla 12 se encuentra el concentrado de los datos, así como los ahorros generados mediante el proyecto. Estos cálculos se hicieron considerando las partes por millón de productos defectuosos antes y después del proyecto obtenidos en sus respectivos estudios de capacidad de proceso. El resultado obtenido fue una reducción proyectada en los costos de $\$ 129,279.73$ dólares estadounidenses por año.

Tabla12: Beneficios obtenidos (en dólares estadounidenses)

\begin{tabular}{lrr}
\hline & Antes del proyecto & Después del proyecto \\
\hline Producción mensual & $1^{\prime} 170,000$ & $1^{\prime} 170,000$ \\
Producción anual & $14^{\prime} 040,000$ & $14{ }^{\prime} 040,000$ \\
PPM & $46,046.15$ & 6.36 \\
Total de defectos anual & $646,487.95$ & 89.29 \\
Costo anual por baja de resistencia & $129,297.59$ & 17.86 \\
\hline \multicolumn{2}{c}{ Ahorro después del proyecto seis sigma } & US\$129,279.73 \\
\hline
\end{tabular}

Además, mediante el concentrado presentado en la Tabla 13 se puede apreciar que la resistencia a la prueba de jalón se aumentó de 9.94 a 17.11 libras lo cual representa un aumento del $72 \%$ 
aproximadamente para esta característica de calidad; la desviación estándar se mantuvo relativamente sin cambio, no obstante las partes por millón si se redujeron de 46,046.15 a 6.36, pasando de un nivel de sigma de 3.18 a 5.86, con un aumento considerable en el Cpk de 0.56 a 1.45, superior al 1.33 fijado como objetivo al inicio del proyecto, como consecuencia del gran aumento presentado en la media de la resistencia a la prueba de jalón del producto. Cabe señalar que se incrementó la satisfacción de los clientes de este producto, reflejándose en la desaparición de retornos por esta causa.

Tabla13: Concentrado de los indicadores de cada uno de los procesos analizados

\begin{tabular}{lcc}
\hline Indicador & Ajuste propuesto & Ajuste inicial \\
\hline Media & 17.11 & 9.94 \\
Desviación estándar & 2.77 & 2.93 \\
Cpk & 1.45 & 0.56 \\
PPM & 6.36 & $46,046.15$ \\
Nivel de sigma & 5.86 & 3.18 \\
\hline
\end{tabular}

\section{CONCLUSIONES}

Mediante la elaboración de este proyecto se pudo demostrar a la empresa la importancia de emprender mejoras en sus procesos basándose en técnicas estadísticas, siguiendo la metodología seis sigma, ya que anteriormente las mejoras se hacían a prueba y error o recolectando una gran cantidad de muestras, de las cuales solo se analizaban su media y desviación estándar a simple vista, sin ninguna prueba estadística, por lo que las conclusiones no eran adecuadas y además los costos de estas pruebas resultaban demasiado elevados ya que utilizaban tamaños de muestra incluso hasta de dos mil unidades.

Otro aspecto importante es que para implementar la mejora, no fue necesaria ninguna inversión, sino solamente controlar el ajuste de cada uno de los factores en los niveles establecidos. Antes de este proyecto no existía una forma fija de operar el proceso y éste se modificaba dependiendo del punto de vista o criterio de los operadores o del supervisor de línea. Los resultados obtenidos en éste proyecto pretenden servir de estímulo a la empresa para el desarrollo de futuros proyectos y mejora de sus procesos mediante la aplicación de la metodología seis sigma.

\section{NOMENCLATURA}

Símbolo Descripción

y) Representa el valor esperado de respuesta

$\bar{y} \quad$ Representa la media general del experimento

$\bar{A}_{1} \quad$ Representa el componente medio del llenado de epóxico al 100\%

$\bar{B}_{2} \quad$ Representa el componente medio de la presión de aplicación en el rango de 50 - 60 psi

$\bar{C}_{2} \quad$ Representa el componente medio de la altura del dado ajustador en altura baja

\section{REFERENCIAS}

Antony, J. y J. Antony; Teaching the Taguchi method to industrial engineers, Work Study: 50(4), 141149 (2001).

AIAG-Automotive Industry Action Group; Measurement Systems Analysis, $3^{\mathrm{a}}$ edición, Southfield, MI: Automotive Industry Action Group (2002).

Bañuelas, R. y J. Antony; Six sigma or design for six sigma?, The TQM Magazine: 16(4), 250-63 (2004). 
Cesarone, J.; The power of Taguchi, Institute of Industrial Engineers Solutions: 33(11), 36-40 (2001).

De la Vara, R.; Análisis y diseño de experimentos, $1^{\text {a }}$ edición, Mc Graw Hill, México, D.F. (2004).

Escalante, E.; Seis sigma: Metodología y técnica, $1^{\text {a }}$ edición, 17 - 21, Limusa, México, D.F. (2003).

Goh, T.N.; Taguchi methods: some technical, cultural and pedagogical perspectives, Quality and Reliability Engineering International: 9, 185-202 (1993).

Henderson, G.R.; Six sigma: Quality Improvement with MINITAB, 1ª edición, Wiley, England (2006).

Jong, W., S. Chen, H. Tsai y C. Chiu; The Geometrical Effects of Bumps on the Fatigue Life of Flipchip Packages by Taguchi Method, Journal of Reinforced Plastics and Composites: 25(1), 99-114 (2006).

Li, M.C., A. Al-Refaie y C. Yang; DMAIC Approach to Improve the Capability of SMT Solder Printing Process, IEEE on Electronics Packaging Manufacturing: 31(2), (2008).

Li, Y.Q., S. Cui, X.Y. Ruan y D.J. Zhang; Application of six sigma Robust Optimization in Sheet Metal Forming, American Institute of Physics: A, 819-824 (2005).

Palanikumar, K; Cutting Parameters Optimization for Surface Roughness in Machining of GFRP Composites using Taguchi's Method, Journal of Reinforced Plastics and Composites: 25(16), 17391751 (2006).

Wang, D. y otros cuatro autores; Note: Determination of Nattokinase Production Condition Using Taguchi Parameter Desing, Food Science and Technology International: 12(3), 215-220 (2006).

Xydas, N. y otros cuatro autores; Dynamic Taguchi Methods and Parameter Design as Applied in Barcode Scanning and Scanners, Concurrent Engineering: Research and Applications: 13(1), 69-80 (2005). 